\title{
Rhinovirus infection and healthcare utilisation in prematurely born infants
}

\author{
Simon B. Drysdale1, Mireia Alcazar-Paris ${ }^{1}$, Theresa Wilson'1, Melvyn Smith², \\ Mark Zuckerman², Simon Broughton', Gerrard F. Rafferty', Janet L. Peacock ${ }^{3}$, \\ Sebastian L. Johnston ${ }^{4}$ and Anne Greenough ${ }^{1}$
}

Affiliations: 'Division of Asthma, Allergy and Lung Biology, MRC-Asthma UK Centre in Allergic Mechanisms of Asthma, King's College London, London, ${ }^{2}$ South London Specialist Virology Centre, King's College Hospital, London, ${ }^{3}$ Division of Health and Social Care Research, King's College London, London, and ${ }^{4}$ Dept of Respiratory Medicine, National Heart and Lung Institute, Imperial College London, London, UK.

Correspondence: A. Greenough, Neonatal Intensive Care Centre, King's College Hospital, Denmark Hill, London SE5 9RS, UK. E-mail: anne.greenoughakcl.ac.uk

ABSTRACT Our aim was to determine whether rhinovirus (RV) lower respiratory tract infections (LRTIs) in prematurely born infants increase health-related cost of care during infancy.

153 infants born at $<36$ weeks of gestation were prospectively followed to 1 year. Cost of care was calculated from the National Health Service reference costing scheme and healthcare utilisation determined by examining hospital/general practitioner records.

20 infants developed RV LRTIs (RV group), 17 respiratory syncytial virus (RSV) LRTIs (RSV group), 12 both RV and RSV LRTIs (RV/RSV group) and 74 had no LRTI (no LRTI group). Compared with the no LRTI group, the RV/RSV LRTI group had the greatest increase in adjusted mean cost (difference GBP 5769), followed by the RV LRTI group (difference GBP 278) and, finally, the RSV LRTI group (difference GBP 172) $(\mathrm{p}=0.045)$. The RV group had more outpatient $(\mathrm{p}<0.05)$ and respiratory-related general practitioner $(\mathrm{p}<0.05)$ attendances, more wheezed at follow-up $(\mathrm{p}<0.001)$ than the no LRTI group and more had respiratory-related outpatient attendances than the RSV LRTI group $(\mathrm{p}<0.05)$.

We conclude that RV LRTIs were associated with increased health-related cost of care during infancy; our results suggest that the RV group compared with the RSV group suffered greater chronic respiratory morbidity.

@ERSpublications

Rhinovirus LRTIs in prematurely born infants increases health-related cost of care during infancy http://ow.ly/mxyZH

Received: July 162012 | Accepted after revision: Feb 092013 | First published online: April 052013

Support statement: Funding was provided by the MRC Grant Centre (G1000758). S.B. Drysdale was supported by the National Institute for Health Research (NIHR) Biomedical Research Centre at Guy's and St Thomas' NHS Foundation Trust/King's College London. S.L. Johnston is supported by a Chair from Asthma UK (CHIISJ). The research nurses were funded by Abbott Laboratories who market Palivizumab.

Conflict of interest: Disclosures can be found alongside the online version of this article at www.erj.ersjournals.com Copyright @ERS 2013 


\section{Introduction}

Respiratory syncytial virus (RSV) lower respiratory tract infections (LRTIs) are associated with increased chronic respiratory morbidity and healthcare utilisation in prematurely born infants who did [1] or did not $[2,3]$ develop bronchopulmonary dysplasia (BPD). Amongst those who had had BPD, healthcare utilisation and the related cost of care were increased up to 7 years of age and lung function was lower than that of controls at 10 years of age [1]. In term-born children, rhinovirus (RV) infection is also associated with chronic respiratory morbidity [4-8]. There is some evidence that RV infection may also have long-term adverse effects on prematurely born infants, in that eight prematurely born infants with BPD who developed RV LRTIs subsequently had a sustained worsening of their clinical status, requiring the addition of new therapies for prolonged periods of time [9]. As a consequence, we hypothesised that prematurely born infants with or without BPD who suffered an RV LRTI would suffer increased healthcare utilisation in infancy and have greater health-related cost of care. The aim of this study was to test that hypothesis. In addition, we wished to determine whether the magnitude of any increase in healthcare utilisation and the health-related cost of care were similar to those associated with RSV LRTI.

\section{Materials and methods}

Study subjects

Infants born at $<36$ weeks of gestational age in 2008 or 2009 were eligible for entry into the study if they were born prior to the onset of the RSV season. The RSV season was defined as October 1 to March 31, consistent with the UK experience. Consecutive infants, including twins and triplets, who lived within the King's College Hospital NHS Foundation Trust $(\mathrm{KCH})$ catchment area, and whose parents gave informed written consent, were recruited. The majority of the infants were born at $\mathrm{KCH}$; the others had been born elsewhere because of a lack of maternity beds or neonatal intensive care unit cots at the time of delivery, but were transferred back to $\mathrm{KCH}$ for their ongoing neonatal care as soon as a cot became available. Infants were recruited either from the neonatal unit or postnatal ward. The study was approved by the research ethics committee of King's College Hospital NHS Trust.

\section{Study design and methods}

Following neonatal unit discharge, infants were followed prospectively until 1 year of corrected age. The parents were asked to contact the research team when their infant was symptomatic with signs consistent with an LRTI, that is, cough, wheeze, and/or shortness of breath. In addition, parents were telephoned every 2 weeks by researchers until the infants were 1 year of corrected age to ascertain whether their infant had been or was symptomatic. A researcher visited the home on every occasion that an infant had an LRTI and a nasopharyngeal aspirate (NPA) was obtained if the LRTI was confirmed by the researcher. NPAs were obtained on each occasion an infant was admitted with an NPA. Real-time reverse transcriptase polymerase chain reaction (PCR) was performed on the NPAs for nine viruses (rhinovirus, RSV A and B, human metapneumovirus, influenza $A$ and $B$, and parainfluenza 1-3) in three multiplexes with a monoplex RNA internal control as previously described [10]. Adenovirus (DNA virus) was tested by real-time reverse transcriptase PCR in monoplex with a DNA internal control (also a monoplex) [10]. In addition, another multiplex, including an MS2 phage internal control, was developed using previously published primers and probes [11-14] that tested for enterovirus, parechovirus and human bocavirus.

Hospital records were examined to identify admissions, accident and emergency and outpatient attendances and all medications prescribed. General practitioner (GP) records were examined to identify any hospital readmissions, accident and emergency attendances, the number of outpatient hospital appointments, the number of GP attendances and referrals to community support services and all medication prescribed. Regarding healthcare utilisation, all visits to practice nurses or routine visits to health visitors, for example, for immunisations, were not recorded as these were considered usual care for infants. Parents completed a respiratory diary card for 1 month when their infant was 11 months of corrected age and a respiratory health-related questionnaire about their infant when the infant was 1 year of corrected age. Parents were asked to record on a daily basis on the respiratory diary cards whether their infant coughed, wheezed or used any "respiratory" medications (inhalers, oral steroids or antibiotics). To calculate the cost of care, the National Health Service reference costing scheme was used; this gives national average costs for in- and outpatient hospital and GP attendances. For admissions, the number of days for each admission was multiplied by the national average cost for the diagnosis leading to admission.

\section{Analysis}

The infants were divided into four groups: 1) infants who never had a symptomatic LRTI (no LRTI); 2) infants who had at least one LRTI from which RV was detected from the NPA (RV LRTI); 3) infants who 
had at least one LRTI from which RSV was detected from the NPA (RSV LRTI); and 4) infants who had LRTI(s) with RV and RSV detected from NPA(s) (RV/RSV LRTI).

The results of infants who had other viral LRTIs, but not an RV or RSV LRTI, or had a symptomatic LRTI, but no virus was detected were excluded from the analysis.

\section{Statistical analysis}

Baseline factors were compared across the four groups using the Kruskal-Wallis test with post hoc tests adjusted for multiple comparisons. The cost data were summarised using means rather than medians to preserve the total sum of costs [15]. Baseline factors were compared across the four groups using the Kruskal-Wallis test with post hoc tests adjusted for multiple comparisons. To analyse the cost data, the approach advocated by BARBER and THOMPSON [16] was followed, fitting a generalised linear model with a gamma distribution and identity link for the total cost data, and a Poisson distribution with robust standard error and identity link for the respiratory cost data. In each case, models were chosen to give estimates as mean costs in a model with deviance residuals close to the normal distribution, as required by the method. In order to account for any differences in the demographics of the three groups, an adjusted analysis was performed with adjustment, first, for birthweight, gestational age, antenatal steroid use and surfactant, and, secondly, for those variables plus BPD. Principal components analysis was first used to reduce the birthweight, gestational age, antenatal steroid use and surfactant data to two principal components that explained almost $\sim 80 \%$ of the total variability in those factors. Those two components were then used as covariates in a further generalised linear model to obtain adjusted estimates. Adjustment using a generalised linear model was not possible for the respiratory data as the majority of the "no LRTI" group had zero costs and so the model was unstable [17]. This is not a statistical power issue, but a consequence of the dominance of nil costs in the no LRTI group, as would be expected.

\section{Results}

251 infants were eligible for inclusion into the study (fig. 1). The 153 (84 male) infants who completed the study had a median gestational age of 34 (range 23-35) weeks and a birthweight of 1890 (range 5343610) g. 20 infants developed RV LRTIs, 17 developed RSV LRTIs, 12 developed both RV/RSV LRTIs and 74 infants had no LRTI. 30 infants had other viral or viral negative LRTIs and their results were excluded from the analysis. Of the 12 infants developing both RV/RSV LRTIs, nine had RV or RSV detected during two separate LRTIs and three infants had detection of RV and RSV during one LRTI. The RV LRTI group had a median of two LRTIs, the RSV LRTI group a median of one LRTI and the RV/RSV LRTI group a median of two LRTIs $(\mathrm{p}<0.001$ across the three groups with post hoc analysis showing no significant difference between the RV and the RV/RSV LRTI groups ( $p=0.95)$, but significant differences between both those groups and the RSV LRTI group $(\mathrm{p}<0.01$ and $\mathrm{p}<0.01$, respectively)).

\section{Demographics}

The significant differences in the demographics (table 1) between the four groups were in gestational age (but there were no significant differences on post hoc analysis), birthweight (the RV LRTI group was significantly lighter at birth than the no LRTI group; $\mathrm{p}<0.05$ ), antenatal steroid use (a significantly greater proportion of the mothers in the RSV group had received antenatal steroids than the no LRTI group; $\mathrm{p}<0.05$ ), surfactant use (a greater proportion of the RV/RSV LRTI group had more surfactant than either the RSV LRTI $(\mathrm{p}<0.05)$ or the no LRTI groups $(\mathrm{p}<0.05)$ ), BPD (but there were no significant differences in post hoc analysis) and palivizumab use (a significantly greater proportion of the RV LRTI group had received palivizumab than the no LRTI group; $\mathrm{p}<0.05$ ).

\section{Healthcare utilisation}

Nine (12\%) infants of the no LRTI group had hospital admissions (all for nonrespiratory causes), two (10\%) of the RV LRTI group had hospital admissions (one for an RV LRTI and one for a nonrespiratory cause), seven (41\%) of the RSV LRTI group had hospital admissions (five for RSV LRTI, one for another viral LRTI and one for a nonrespiratory cause) and six (50\%) of the RV/RSV LRTI group had hospital admissions (two for an RV/RSV LRTI, three for a RSV LRTI and one for an RV LRTI).

The RV LRTI group had more total $(\mathrm{p}<0.05)$ and respiratory-related $(\mathrm{p}<0.05)$ outpatient attendances and GP respiratory-related attendances $(\mathrm{p}<0.05)$ than the no LRTI group and more respiratory-related outpatient attendances than the RSV LRTI group $(\mathrm{p}<0.05)$. The RSV LRTI group had more total $(\mathrm{p}<0.05)$ and respiratory-related $(\mathrm{p}<0.05)$ hospitalisations than the no LRTI group and more total $(\mathrm{p}<0.05)$ and respiratory-related $(\mathrm{p}<0.05)$ hospitalisations than the RV LRTI group. The RSV LRTI group had more total $(\mathrm{p}<0.05)$ and respiratory-related $(\mathrm{p}<0.05)$ accident and emergency attendances than the no LRTI group (table 2). The RV/RSV group had more total and respiratory-related hospitalisations than both the 


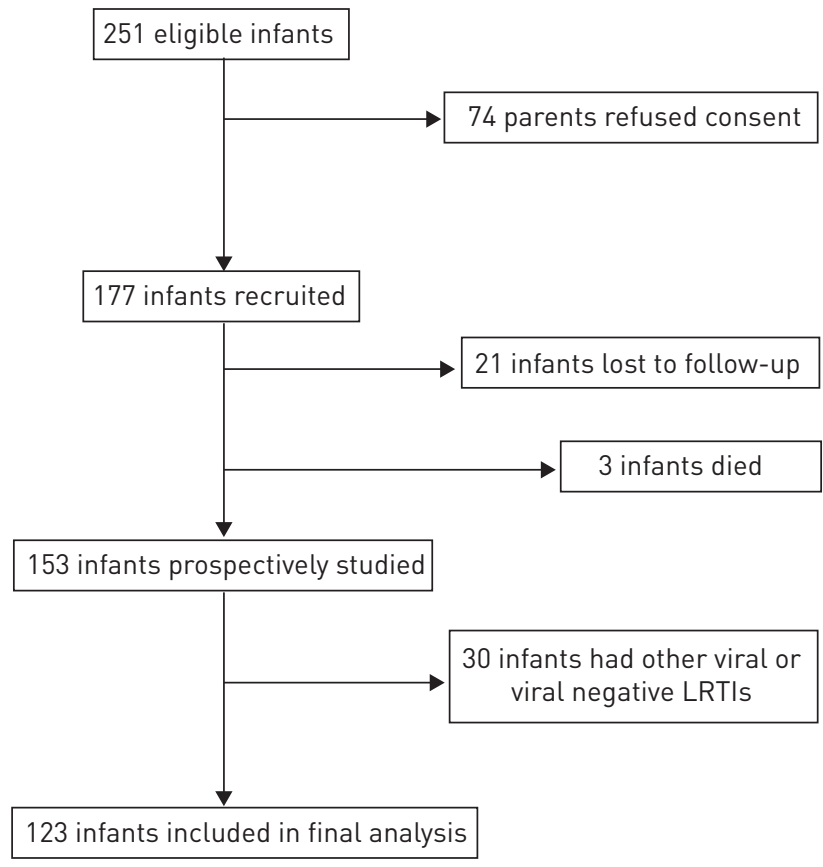

FIGURE 1 Consort diagram of recruitment. LRTI: lower respiratory tract infection.

no LRTI $(\mathrm{p}<0.05)$ and RV LRTI $(\mathrm{p}<0.05)$ groups. The RV/RSV group had more total $(\mathrm{p}<0.05)$ and respiratory-related $(\mathrm{p}<0.05)$ accident and emergency attendances than the no LRTI group (table 2$)$. The RV/RSV LRTI group had more total outpatient attendances than the no LRTI group $(\mathrm{p}<0.05)$ (table 2).

Diary card and respiratory healthcare data

Analysis of the diary card data highlighted that the RV/RSV LRTI group had more days of inhaler use than any of the other three groups $(\mathrm{p}<0.05, \mathrm{p}<0.05$ and $\mathrm{p}<0.05$, respectively) (table 3 ). Analysis of the

\section{TABLE 1 Demographic data according to lower respiratory tract infection (LRTI) status}

\begin{tabular}{|c|c|c|c|c|c|}
\hline & No LRTI & RV LRTI & RSV LRTI & RV/RSV LRTI & Overall p-value \\
\hline Subjects & 74 & 20 & 17 & 12 & \\
\hline Gestational age weeks & $34.1(25.7-35.9)$ & $32.9(27.4-35.7)$ & $32.6(28.9-35.6)$ & 32.1 (23.0-35.9) & 0.044 \\
\hline Birthweight $\mathbf{g}$ & 2070 (895-3610) & $1558(670-2512)$ & $1756(1080-2650)$ & 1595 (610-2546) & 0.002 \\
\hline Males & $39(53)$ & $9(45)$ & $9(53)$ & $5(42)$ & 0.85 \\
\hline Ethnicity & & & & & 0.043 \\
\hline Caucasian & $28(38)$ & $2(10)$ & $3(18)$ & $2(17)$ & \\
\hline Black Caribbean & $19(26)$ & $2(10)$ & $5(29)$ & $2(17)$ & \\
\hline Black African & $12(16)$ & $4(20)$ & $5(29)$ & $2(17)$ & \\
\hline Asian & $3(4)$ & $2(10)$ & $1(6)$ & $0(0)$ & \\
\hline Hispanic & $1(1)$ & $0(0)$ & $0(0)$ & $1(8)$ & \\
\hline Mixed race & $11(15)$ & $10(50)$ & $3(18)$ & $5(41)$ & \\
\hline Antenatal smoking ${ }^{\#}$ & $11(15)$ & $4(20)$ & $2(12)$ & $2(17)$ & 0.91 \\
\hline Antenatal steroids & $40(54)$ & $16(80)$ & $16(94)$ & $8(67)$ & 0.014 \\
\hline Surfactant & $11(15)$ & $7(35)$ & $1(6)$ & $6(50)$ & 0.005 \\
\hline Duration of ventilation days & $0(0-82)$ & $1(0-103)$ & $1(0-17)$ & $1.5(0-81)$ & 0.39 \\
\hline Bronchopulmonary dysplasia & $4(5)$ & $5(25)$ & $0(0)$ & $3(25)$ & 0.008 \\
\hline Family history of atopy & $52(70)$ & $13(65)$ & $8(47)$ & $7(58)$ & 0.32 \\
\hline Breastfeeding & $62(84)$ & 15 (75) & $11(65)$ & $8(67)$ & 0.20 \\
\hline Eczema & $20(28)$ & $3(16)$ & $2(12)$ & $2(17)$ & 0.45 \\
\hline Daycare attendance & $2(3)$ & $0(0)$ & $1(6)$ & $1(8)$ & 0.52 \\
\hline Number of siblings & $1(0-5)$ & $1(0-5)$ & $1(0-4)$ & $1(0-4)$ & 0.91 \\
\hline Palivizumab & $0(0)$ & $3(15)$ & $0(0)$ & $1(8)$ & 0.005 \\
\hline
\end{tabular}

Data are presented as $\mathrm{n}$, median (range) or $\mathrm{n}(\%)$, unless otherwise stated. RV: rhinovirus; RSV: respiratory syncytial virus. ${ }^{\#}$ : all mothers who smoked antenatally also smoked postnatally; ${ }^{\natural}$ : a family history of atopy was defined as a first degree relative (parent or sibling) with asthma, eczema, hay fever or an allergy. 
TABLE 2 Healthcare utilisation to 1 year corrected age according to lower respiratory tract infection (LRTI) status

\begin{tabular}{|c|c|c|c|c|c|}
\hline Admissions & 74 & 20 & 17 & 12 & \\
\hline Respiratory & $0(0)(0-0)$ & $0(0.1)(0-1)$ & $0(0.4)(0-1)$ & $0(1.3)(0-11)$ & $<0.001$ \\
\hline \multicolumn{6}{|c|}{$\begin{array}{l}\text { Accident and emergency } \\
\text { attendances }\end{array}$} \\
\hline Total & $0(0.7)(0-6)$ & $1(1.2)(0-5)$ & $1(1.6)(0-8)$ & $1(2.5)(0-14)$ & 0.015 \\
\hline Total & $2(3.3)(0-14)$ & $5(6.9)(0-18)$ & $4(4.0)(0-10)$ & 5 (7.3) (1-15) & 0.002 \\
\hline Respiratory & $0(0)(0-0)$ & $0(1.1)(0-9)$ & $0(0)(0-0)$ & $0(0.8)(0-8)$ & $<0.001$ \\
\hline \multicolumn{6}{|l|}{ GP attendances } \\
\hline Total & $5(5.3)(0-20)$ & $6(6.8)(1-15)$ & $5(6.1)(2-14)$ & $9(8.3)(3-15)$ & 0.069 \\
\hline Respiratory & $0(0.4)(0-2)$ & $1(1.4)(0-4)$ & $1(0.8)(0-3)$ & $1(1.8)(0-6)$ & 0.002 \\
\hline
\end{tabular}

Data are presented as $\mathrm{n}$ or median (mean) (range), unless otherwise stated. RV: rhinovirus; RSV: respiratory syncytial virus; GP: general practitioner.

respiratory health-related questionnaire data (table 4) demonstrated that a greater proportion of the RV LRTI group wheezed than the no LRTI group $(\mathrm{p}<0.001)$. Greater proportions of the RV/RSV LRTI group wheezed $(p<0.001)$ and used bronchodilators $(p<0.01)$ or preventers $(p<0.001)$ than the no LRTI group (table 4). A greater proportion of the RV/RSV LRTI group used preventers compared with the RSV LRTI group $(\mathrm{p}<0.001)$ (table 4$)$.

\section{Health-related cost of care}

There was a significant difference overall across the four groups in the mean costs for outpatient attendances, GP respiratory attendances and medication (table 5). The costs for outpatient attendances were greater in the RV LRTI group compared with the no LRTI group $(\mathrm{p}<0.05)$ and compared with the RSV LRTI group $(\mathrm{p}<0.05)$. There were no significant differences between the four groups on post hoc analysis for the costs for GP respiratory attendances or medications.

Compared with the no LRTI group, the RV/RSV LRTI group had the highest mean cost (difference GBP7035), followed by the RV LRTI group (difference GBP1086) and, finally, the RSV LRTI group (difference GBP678). These differences were reduced after adjusting for birthweight, gestational age, antenatal steroid and surfactant use, but overall the differences remained statistically significant. In addition, further adjustment for BPD reduced the differences slightly more, but the overall differences in costs between groups remained significant $(\mathrm{p}=0.045)$ (table 6). The health-related cost of care of the RV LRTI group was similar to that of the RSV LRTI group $(\mathrm{p}=0.83)$ (table 6). The ordering of costs was the same for total respiratory costs and the differences in mean costs were statistically significant $(\mathrm{p}=0.003)$ (table 7). Adjustment for neonatal factors for total respiratory costs was not possible as models with possible confounders would not converge.

\section{Discussion}

We have demonstrated that prematurely born infants developing RV LRTIs had increased health-related cost of care during infancy compared with infants who did not develop an LRTI. Our results demonstrating

TABLE 3 Diary card data at 1 year corrected age

\begin{tabular}{|c|c|c|c|c|c|}
\hline & No LRTI & RV LRTI & RSV LRTI & RV/RSV LRTI & p-value \\
\hline Subjects ${ }^{\#}$ & 49 & 16 & 11 & 9 & \\
\hline Days of cough & $0(3.3)(0-31)$ & $0.5(4.9)(0-18)$ & $0(6.5)(0-23)$ & $3(7.8)(0-30)$ & 0.40 \\
\hline Days using antibiotics & $0(0)(0-2)$ & $0(0)(0-0)$ & $0(0.6)(0-7)$ & $0(1.1)(0-5)$ & 0.042 \\
\hline Days using inhalers & $0(0)(0-0)$ & $0(1.9)(0-30)$ & $0(0)(0-0)$ & $0(4.3)(0-30)$ & $<0.001$ \\
\hline
\end{tabular}

Data are presented as $n$ or median (mean) (range), unless otherwise stated. LRTI: lower respiratory tract infection; RV: rhinovirus; RSV: respiratory syncytial virus. ${ }^{\#}$ : not all patients completed the diary card. 
TABLE 4 Respiratory health-related questionnaire data

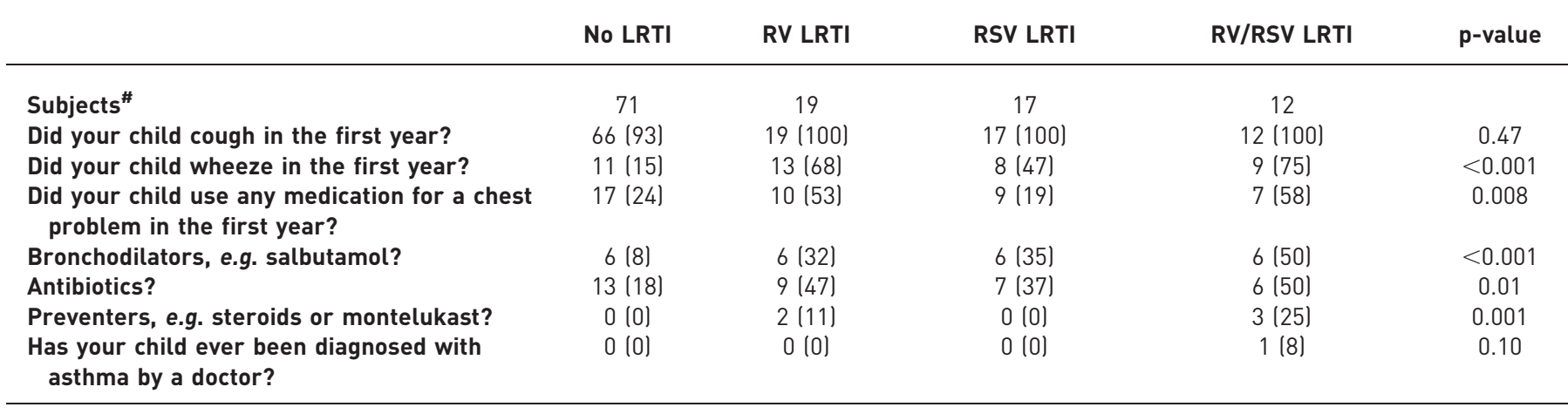

Data are presented as $\mathrm{n}$ or $\mathrm{n}(\%)$, unless otherwise stated. LRTI: lower respiratory tract infection; RV: rhinovirus; RSV: respiratory syncytial virus. \#: not all parents completed the questionnaire and those reported responded yes to the question.

the RV LRTI group suffered chronic respiratory morbidity are in keeping with previous findings in eight prematurely born infants [9]. In that series, however, all of the infants were born very prematurely, had had BPD and were hospitalised with the RV LRTI [9]. Whereas, the 20 infants in the RV group presently studied were born at a higher gestational age, only $25 \%$ had had BPD and only one of the 20 infants had been hospitalised with the RV LRTI.

The RV/RSV group, like the RSV group, had significantly more admissions and accident and emergency attendances than the no LRTI group. It has been previously reported that infants who develop a dual infection either with RSV and human metapneumovirus [18] or RSV and RV [19] are more likely to develop a severe LRTI, as proven by requirement for a paediatric intensive care unit admission. In this study, however, only three of the infants had RV and RSV detected during the same LRTI. The higher admission rate of the RV/RSV group may reflect a functional predisposition to severe viral LRTIs. We have previously shown that very prematurely born infants who developed an RSV LRTI and subsequent chronic respiratory morbidity had significantly worse pre-morbid lung function [20]. In addition, among infants with a wider range of gestational ages, those who required hospitalisation for an RSV LRTI had significantly poorer pre-morbid lung function [21]. In infants born at term, poorer pre-morbid lung function, that is, a higher resistance of the respiratory system in the first 2 months after birth, was associated with an increased

TABLE 5 Costs of care (GBP) to 1 year corrected age according to lower respiratory tract infection (LRTI) status

\begin{tabular}{|c|c|c|c|c|c|}
\hline & No LRTI & RV LRTI & RSV LRTI & RV/RSV LRTI & Overall p-value \\
\hline Subjects & 74 & 20 & 17 & 12 & \\
\hline \multicolumn{6}{|l|}{ Admission } \\
\hline Total cost & $188 \pm 654$ & $139 \pm 450$ & $665 \pm 1006$ & $5771 \pm 10168$ & 0.51 \\
\hline Respiratory cost & $0 \pm 0$ & $48 \pm 214$ & $337 \pm 627$ & $3470 \pm 7740$ & 0.83 \\
\hline \multicolumn{6}{|c|}{ Accident and emergency } \\
\hline Total cost & $59 \pm 100$ & $109 \pm 148$ & $158 \pm 228$ & $286 \pm 509$ & 0.09 \\
\hline Respiratory cost & $5 \pm 33$ & $35 \pm 116$ & $77 \pm 113$ & $247 \pm 486$ & 0.73 \\
\hline \multicolumn{6}{|l|}{ Outpatient } \\
\hline Total cost & $445 \pm 418$ & $1402 \pm 1689$ & $522 \pm 321$ & $1227 \pm 1317$ & 0.002 \\
\hline Respiratory cost & $0 \pm 0$ & $605 \pm 1444$ & $0 \pm 0$ & $362 \pm 1210$ & Unable to calculate $^{\#}$ \\
\hline \multicolumn{6}{|l|}{ GP } \\
\hline Total cost & $287 \pm 252$ & $439 \pm 380$ & $313 \pm 196$ & $731 \pm 931$ & 0.05 \\
\hline Respiratory cost & $16 \pm 25$ & $55 \pm 52$ & $32 \pm 37$ & $75 \pm 95$ & 0.02 \\
\hline \multicolumn{6}{|l|}{ Medication } \\
\hline Total cost & $109 \pm 173$ & $672 \pm 1218$ & $104 \pm 150$ & $719 \pm 1334$ & 0.04 \\
\hline Respiratory cost & $2 \pm 3$ & $484 \pm 1195$ & $3 \pm 5$ & $286 \pm 963$ & 0.09 \\
\hline \multicolumn{6}{|l|}{ Overall } \\
\hline Total cost & $980 \pm 958$ & $2067 \pm 1794$ & $1658 \pm 1299$ & $8015 \pm 12072$ & 0.001 \\
\hline Respiratory cost & $27 \pm 78$ & $740 \pm 1477$ & $446 \pm 691$ & $4153 \pm 9335$ & 0.003 \\
\hline
\end{tabular}

Data are presented as $n$ or mean \pm SD, unless otherwise stated. RV: rhinovirus; RSV: respiratory syncytial virus; GP: general practitioner.

\#: generalised linear model did not converge and so the $p$-value was unobtainable. 
TABLE 6 Unadjusted and adjusted mean total costs (GBP) according to lower respiratory tract infection (LRTI) status

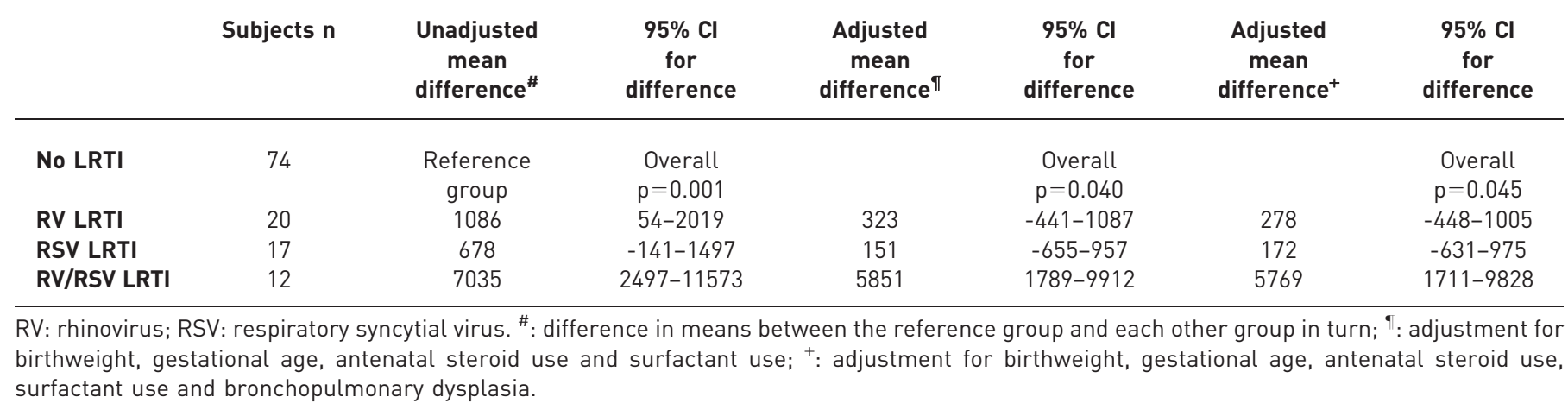

risk of the occurrence and duration of RV-associated wheeze during infancy [22]. It is thus possible that poorer pre-morbid lung function might explain the chronic respiratory morbidity of our RV group.

In infants born at term, RV seems to preferentially affect the lower airways, causing bronchiolitis in atopic children prone to wheezing [23-25]. It has been suggested that the reduced interferon (IFN)- $\gamma$ responses in infancy in children with atopy may partly explain why atopy is a risk factor for human rhinovirus-induced wheezing [5]. Interferon responses in early life are inversely associated with the severity of viral respiratory illnesses [26]. In addition, infants with low ex vivo IFN- $\gamma$ responses in early life are more likely to have frequent viral respiratory illnesses, including those associated with wheezing [27]. In this study, we performed skin prick tests on the infants, despite our experience being that parents of prematurely born infants frequently refuse skin prick testing in a research setting, as do prematurely born children [1]. We did not, however, see any significant differences between our groups in a family history of atopy or the proportion of infants who had eczema. Whether atopy does predispose prematurely born infants to RV LRTIs needs further investigation.

Our study has a number of strengths and some limitations. We prospectively followed a large cohort of prematurely born infants from birth to 1 year of corrected age. We were able to investigate symptomatic LRTIs, not only in hospitalised infants, but also in those with LRTIs in the community. This is important, as we have previously demonstrated that infants with RSV LRTI not requiring hospitalisation also suffer increased respiratory morbidity [2]. In addition, the NPAs were tested by real-time PCR multiple assay that had the advantage of high sensitivity to detect a wide range of respiratory viruses [28, 29]. A limitation of our study, however, is that we only obtained NPAs when the infants were symptomatic and thus we cannot comment as to whether asymptomatic LRTIs increase healthcare utilisation at follow-up. Both the RV LRTI and the RV/RSV LRTI groups had a median of two LRTIs, but the RSV LRTI group had a median of one LRTI, which might have influenced differences in the cost of care. The RV LRTI and RSV LRTI groups, however, had similar costs of care, but the type of healthcare utilisation that resulted in the increased costs of care compared with the no LRTI group differed between the two groups. Infants were recruited before the RSV season to ensure all infants were exposed to a whole RSV season. All infants, however, were followed for 1 year so that seasonal variations between viruses would not affect our results. Our groups differed significantly in respect to certain of their demographics; the no LRTI group were more mature at birth, of higher birthweight and were less likely to have received antenatal steroids. Our findings of

TABLE 7 Unadjusted mean difference in respiratory costs (GBP) according to lower respiratory tract infection (LRTI) status

Subjects $\mathbf{n}$

\begin{tabular}{lcc}
\hline No LRTI & 74 & Reference group \\
RV LRTI & 20 & 713 \\
RSV LRTI & 17 & 418 \\
RV/RSV LRTI & 12 & 4125 \\
\hline
\end{tabular}

RV: rhinovirus; RSV: respiratory syncytial virus. " : difference in means between the reference group and each other group in turn. An adjusted model could not be fitted due to convergence problems. 
increased health-related costs of care compared with the no LRTI group, however, remained statistically significant after adjusting for neonatal factors.

In conclusion, RV LRTIs in prematurely born infants were associated with an increased health-related cost of care during infancy. The increased health-related cost of care in the RV LRTI group was due to more outpatient and respiratory-related GP attendances and a greater proportion of the group wheezed at followup, whereas in the RSV LRTI group it was due to more admissions. Those data suggest the RV LRTI group suffered greater chronic respiratory morbidity than the RSV LRTI group.

\section{References}

1 Greenough A, Alexander J, Boit P, et al. School age outcome of hospitalisation with respiratory syncytial virus infection of prematurely born infants. Thorax 2009; 64: 490-495.

2 Broughton S, Roberts A, Fox G, et al. Prospective study of health care utilisation and respiratory morbidity due to RSV infection in prematurely born infants. Thorax 2005; 60: 1039-1044.

3 Broughton S, Sylvester KP, Fox G, et al. Lung function in prematurely born infants following viral lower respiratory tract infections. Pediatr Infect Dis J 2007; 26: 1019-1024.

4 Jackson DJ, Gangnon RE, Evans MD, et al. Wheezing rhinovirus illnesses in early life predict asthma development in high-risk children. Am J Respir Crit Care Med 2008; 178: 667-672.

5 Jartti T, Korppi M. Rhinovirus induced bronchiolitis and asthma development. Pediatr Allergy Immunol 2011; 22: 350-355.

6 Midulla F, Pierangeli A, Cangiano G, et al. Rhinovirus bronchiolitis and recurrent wheezing: 1 year follow up. Eur Respir J 2012; 39: 396-402.

7 Guilbert TW, Singh AM, Danov Z, et al. Decreased lung function after preschool wheezing rhinovirus illnesses in children at risk to develop asthma. J Allergy Clin Immunol 2011; 128: 532-538.

8 Lemanske RF, Jackson DJ, Gangnon RE, et al. Rhinovirus illnesses during infancy predict subsequent childhood wheezing. J Allergy Clin Immunol 2005; 116: 571-577.

9 Chidekel AS, Rosen CL, Bazzy AR. Rhinovirus infection associated with serious lower respiratory illness in patients with bronchopulmonary dysplasia. Pediatr Infect Dis J 1997; 16: 43-47.

10 Auburn H, Zuckerman M, Broughton S, et al. Detection of nine respiratory RNA viruses using three multiplex RTPCR assays incorporating a novel RNA internal control transcript. J Virol Methods 2011; 176: 9-13.

11 Brittain-Long R, Nord S, Olofsson S, et al. Multiplex real-time PCR for detection of respiratory tract infections. J Clin Virology 2008; 41: 53-56.

12 Lu X, Chittaganpitch M, Olsen S, et al. Real-time PCR assays for detection of bocavirus in human specimens. J Clin Microbiology 2006; 44: 3231-3235.

13 Nix WA, Maher K, Johansson ES, et al. Detection of all known parechoviruses by real-time PCR. J Clin Microbiology 2008; 46: 2519-2524.

14 Rolfe KJ, Parmar S, Mururi D, et al. An internally controlled, one-step, real-time RT-PCR assay for norovirus detection and genogrouping. J Clin Virology 2007; 39: 318-321.

15 Thompson SG, Barber JA. How should cost data in pragmatic randomised trials be analysed. BMJ 2000; 320 : 1197-2000.

16 Barber J, Thompson S. Multiple regression of cost data: use of generalised linear models. J Health Serv Res Policy 2004; 9: 197-204

17 Peacock J, Peacock P. The Oxford Handbook of Medical Statistics, Chapter 12. Oxford, Oxford University Press, 2010

18 Semple MG, Cowell A, Dove W, et al. Dual infection of infants by human metapneumovirus and human respiratory syncytial virus is strongly associated with severe bronchiolitis. J Infect Dis 2005; 191: 382-386.

19 Richard N, Komurian-Pradel F, Javouhey E, et al. The impact of dual viral infection in infants admitted to a pediatric intensive care unit associated with severe bronchiolitis. Pediatr Infect Dis J 2008; 27: 213-217.

20 Broughton S, Bhat R, Roberts A, et al. Diminished lung function, RSV infection, and respiratory morbidity in prematurely born infants. Arch Dis Child 2006; 91: 26-30.

21 Drysdale SB, Wilson T, Alcazar M, et al. Lung function prior to viral lower respiratory tract infections in prematurely born infants. Thorax 2011; 66: 468-473.

22 van der Zalm MM, Uiterwaal CS, Wilbrink B, et al. The influence of neonatal lung function on rhinovirus associated wheeze. Am J Respir Crit Care Med 2011; 183: 262-267.

23 Gern JE, Busse WW. Association of rhinovirus infections with asthma. Clin Microbiol Rev 1999; 12: 9-18.

24 Eirnarsson O, Geba GP, Zhu Z, et al. Interleukin-11: stimulation in vivo and in vitro by respiratory viruses and induction of airways hyperresponsiveness. J Clin Invest 1996; 97: 915-924.

25 Gern JE, Vrtis R, Grindle KA, et al. Relationship of upper and lower airway cytokines to outcome of experimental rhinovirus infection. Am J Respir Crit Care Med 2000; 162: 2226-2231.

26 Papadopoulos NG, Stanciu LA, Papi A, et al. A defective type I response to rhinovirus in atopic asthma. Thorax 2002; 57: 328-332.

27 Copenhaver CC, Gern JE, Li Z, et al. Cytokine response patterns, exposure to viruses and respiratory infections in the first year of life. Am J Respir Crit Care Med 2004; 170: 175-180.

28 Kuypers J, Wright N, Ferrenberg J, et al. Comparison of real-time PCR assays with fluorescent-antibody assays for diagnosis of respiratory virus infections in children. J Clin Microbiol 2006; 44: 2382-2388.

29 van de Pol AC, van Loon AM, Wolfs TF, et al. Increased detection of respiratory syncytial virus, influenza viruses, parainfluenza viruses, and adenoviruses with real-time PCR in samples from patients with respiratory symptoms. J Clin Microbiol 2007; 45: 2260-2262. 